\title{
EVALUATION OF PERIPAPILLARY RETINAL NERVE FIBRE LAYER AND CENTRAL MACULAR THICKNESS INADULTS WITH HYPEROPIC ANISOMETROPIC AMBLYOPIA
}

\author{
Tanveer Ahmed, Khizar Niazi, Sobia Usman Shah, Junaid Afsar Khan, Husnain Muhammad Bukhsh*, \\ Muhammad Arslan Ashraf** \\ Combined Military Hospital Lahore/National University of Medical Sciences (NUMS) Pakistan, *Ganga Ram Hospital, Lahore Pakistan, \\ **Mayo Hospital, Lahore Pakistan
}

\begin{abstract}
Objective: To study peripapillary retinal nerve fibre layer (pRNFL) and central macular thickness (CMT) changes in adults with hyperopic anisometropic amblyopia using optical coherence tomography.

Study Design: Cross sectional study.

Place and Duration of Study: Combined Military Hospital, Lahore, Pakistan, from Oct 2019 to Feb 2020.

Methodology: In this study 30 adults, 18-40 years of age were included who presented in our clinic with monocular poor vision. They underwent detailed ophthalmic clinical examination: including corrected and uncorrected distance visual acuity, slit lamp bio-microscopy and fundus examination with 90 diopter lens. After fulfilling criteria of anisometropic amblyopia, central maular and peripapillary retinal nerve fibre layer thickness was measured by using RS-3000 SLO, NIDEK Co, Japan spectral domain optical coherence tomography device and compared the central maular thickness and peripapillary retinal nerve fibre layer thickness in amblyopic and fellow eye of same individual.

Results: The mean change in peripapillary retinal nerve fibre layer thickness in amblyopic eyes, $121.48 \pm 4.90 \mu \mathrm{m}$ and non amblyopic eyes was $112.92 \pm 4.72 \mu \mathrm{m}$ with statistical significance $(p<0.001)$. The mean change in central macular thickness in amblyopic eyes was $198.50 \pm 5.30 \mu \mathrm{m}$ and non amblyopic eyes was $206.80 \pm 3.11 \mu \mathrm{m}$ with statistical significance $(p<0.001)$. There was significant increase in peripapillary retinal nerve fibre layer thickness and significant decrease in central macular thickness on comparing the amblyopic and the fellow eyes of the same patients.

Conclusion: Central macular thickness decreased while the peripapillary retinal nerve fibre layer thickness increased in patients with hyperopic anisometropic amblyopia.
\end{abstract}

Keywords: Anisometropia, Amblyopia, Central macular thickness (CMT), Optical coherence tomography (OCT), Peri-papillary retinal nerve fibre layer (pRNFL).

This is an Open Access article distributed under the terms of the Creative Commons Attribution License (http://creativecommons.org/licenses/by/4.0), which permits unrestricted use, distribution, and reproduction in any medium, provided the original work is properly cited.

\section{INTRODUCTION}

Worldwide the most commonest cause of moderate to severe visual impairment is considered to be refractive errors. In Pakistan it is thought to be about $8.9 \%$ in school going children. Refractive errors are classified as hypermetropia, myopia and astigmatism. Difference in refractive status of both eyes is known as anisometropia ${ }^{1}$. Anisometropia further leads to amblyopia in presence or absence of strabismus. Anisometropia is considered to be most important cause of amblyopia. Mechanism of anisome-tro-

Correspondence: Dr Tanveer Ahmed, Graded Ophthalmologist, Combined Military Hospital Skardu Pakistan

Received: 01 Mar 2020; revised received: 06 Aug 2020; accepted: 18 Aug 2020 pic amblyopia is still not fully known². Monocular reduction of vision is seldom reported by children which later develops amblyopia and affects vision related quality of life. The diagnosis is usually incidental on routine ophthalmic examination or mass community screening 3 .

Amblyopia is a decrease in best corrected visual acuity (BCVA), in the absence of any identifiable pathology of the human optical system ${ }^{4}$. There are multiple types, namely: strabismus, meridonial, ametropic, anisometropic and stimulus deprivational amblyopia ${ }^{5}$. Amongst these, anisometropic amblyopia is least diagnosed early6. Anisometropic amblyopia can be defined as visual acuity in worse eye $<20 / 32$ with difference of 
2 lines as compare to better eye associated with anisometropia. Its $\geq 1.00 \mathrm{D}$ spherical equivalent (SE) for hyperopia, $\geq 3.00 \mathrm{D}$ spherical equivalent (SE) for myopia and $\geq 1.50 \mathrm{D}$ for astigmatism on auto-refractometer 6 . Being asymptomatic and due to an absence of any external ocular manifestations the diagnosis is built mainly on auto-refractometer findings.

Multiple studies have been conducted which has shown a functional and a structural changes in amblyopic eyes ${ }^{7}$. There is strong association of cellular atrophy in lateral geniculate ganglion and amblyopic eyes. Further advances in neuroanatomy and neurophysiology have shown some probability of retinal dysfunction in amblyopia. Now it has been assumed that retina is a motivating region for amblyopia and further studies are being carried out. Alteration in retina in amblyopic eyes is not yet clear. Amblyopia might affects retinal ganglion (RGCs) cells which further cause abnormalties and decrease in RGCs ${ }^{8}$.

Introduction of optical coherence tomography (OCT) in practice of opthalmology, enabled to measure peripapillary retinal nerve fibre layer (pRNFL) and macular thickness. OCT is a non contact and non invasive procedure uses light rays to take $10 \mathrm{~mm}$ thick optical section of tissues through optical refraction. It has good repoducibility for measuring retinal sturctures in vivo. OCT has made possible to carryout study in patients of amblyopia with advantage to measure peripappillary retinal nerve fibre layer(pRNFL) and central macular thickness9.

Studies suggest the possible role of peripapillary retinal nerve fibre layer ( $p R N F L$ ) and central macular thickness (CMT) in the pathogenesis of anisometropic amblyopia, although the results are highly variable ${ }^{10}$. Some studies have shown no changes in pRNFL or CMT, some studies have shown only macular changes, some are the opinion of pRNFL change only and some studies reported changes in both structures i.e CMT and pRNFL in amblyopic versus non amblyopic fellow eyes.
As the definition suggests, amblyopia is a decrease in best corrected visual acuity in absence of any identifiable pathology of human optical system. Thus, the difference in structure between the two eyes logically constitutes sufficient evidence to exclude anisometropia from the aetiology of amblyopia. Limited workis conducted in Pakistan to measure peripapillary retinal nerve fibre (pRNFL) and central macular thickness (CMT) in adults with hyperopic anisometropic amblyopia. There are also conflicting results about peripapillary retinal nerve fibre ( $\mathrm{pRNFL}$ ) and central macular thickness (CMT) measurements in amblyopic eyes warranting the need for carrying out this study in the Pakistani population.

\section{METHODOLOGY}

This cross sectional study was conducted at Combined Military Hospital Lahore Pakistan, from Octobar 2019 to February 2020. Patients selected for study after fulfilling inclusion and exclusion criteria and written informed consent was taken after permission from research review board Combined Military Hospital Lahore, ref no 149/2020. Sampling was done through consecutive non probability sampling. Thirty healthy subjects 1, 2 between 18-40 years of age, diagnosed hyperopic anisometropic amblyopia with best corrected visual acuity of 6/6 in normal eye were included. Sample size was calculated by comparing of two means of RNFL thickness, using statistics and sample size pro. Patients having family history of glaucoma, corneal orretinal dystrophy, previous history of ocular or laser surgery, previous history of trauma, history of diabetic retinopathy and any identifiable pathology in eyes which has been confirmed via a slit lamp examination were excluded. Selected patients fulfilling the criteria underwent ophthalmic clinical examination including corrected and uncorrected distance visual acuity measurements, slit lamp examination, Ophthalmoscopy and measurement of IOP by applanation tonometer. All measurements / examinations were taken by a single person ( $1{ }^{\text {st }}$ author) in order to exclude observer bias. 
Pupils were dilated with one drop of $1 \%$ Cyclopentolate; instilled three times, with gap of 10 minutes, cycloplegic refraction was carried out using Full Auto Ref-Keratometer (Canon RK-F1, U.S.A). Upon confirmation of hyperopic anisometropic amblyopia, pRNFL thickness was measured using Spectral Domain OCT (RS-3000 SLO, NIDEK Co, Japan) in both eyes, After measuring pRNFL thickness, CMT was measured in central $3.5 \mathrm{~mm}$ of the macula, by SD OCT similarly for both eyes. Data was evaluated and analysed using a version of SPSS-21. Shapiro-Wilk test was applied to check normality. Independent sample Mann Whitney U-test was applied as normality test shows significant $p$-value $(p<0.05)$.

\section{RESULTS}

A total number of 30 patients participated in this study. Out of 30 patients $13(43 \%)$ were female and $17(57 \%)$ were male. Mean age of patients was $29 \pm 11$ years (table-I). Mean change in pRNFL thickness in amblyopic eyes were

Table-I: Stratification of Age and gender.

\begin{tabular}{l|c|c|c}
\hline \multirow{2}{*}{$\mathrm{n}(\%)$} & \multicolumn{3}{|c}{ Age } \\
\cline { 2 - 4 } & Female & Male & Total \\
\hline Minimum & $13(43 \%)$ & $17(57 \%)$ & 30 \\
\hline Maximum & 40 & 18 & 18 \\
\hline Mean & 29 & 40 & 40 \\
\hline $\begin{array}{l}\text { Std. } \\
\text { Deviation }\end{array}$ & 11 & 29 & 29.0 \\
\hline
\end{tabular}

central macular thickness in amblyopic eyes were $198.50 \pm 5.309$ and non amblyopic eyes were $206.80 \pm 3.112$ (table-II).

\section{DISCUSSION}

Amblyopia is a decrease in the best corrected visual acuity (BCVA) in absence of any organic cause $^{11}$. Vision disorders which are most common in children arestrabismus, amblyopia and significant refractive error. Amblyopia is the foremost cause of monocular loss of vision and involves about $1-5 \%$ of children worldwide. Normal development of vision occurs ininfancy and is the most critical period for development of eye sight in children and adults in future ${ }^{6}$.

Difference in refractive power of anisometropic amblyopic eyescreates unclear image on retina which further impair visual development. It is thought that anisometropic amblyopia develops secondary to different neuronal mechani$\mathrm{sms}^{12}$. Normal development of occipital cortex occurs during development and maturation phase of the visual system. Any irregularity in this phase causes permanent loss of vision of that eye $^{6}$. Amblyopia isprimarilya disorder of cortex which iscaused byasymmetrical input from the two eyes into the primary visual cortex ${ }^{11}$.

This study comparedcentral macular thickness (CMT) and peripapillary retinal nerve fibre layer (pRNFL) of hyperopic anisometropic amblyopic eyes and non amlyopic fellow eyes of

Table-II: Comparison of peripapillary retinal nerve fibre layer (pRNFL) and central macular thickness (CMT) in amblyopic and non amblyopic eyes.

\begin{tabular}{l|c|c|c|c}
\multirow{2}{*}{ Group } & \multicolumn{2}{|c|}{$\begin{array}{c}\text { Peripapillary Retinal Nerve Fibre Layers } \\
\text { Thickness }\end{array}$} & \multicolumn{2}{c}{ Central Macular Thickness } \\
\cline { 2 - 5 } & Amblyopia & Non-Amblyopic & Amblyopia & Non-Amblyopic \\
\hline $\mathrm{N}$ & 30 & 30 & 30 & 30 \\
\hline Minimum & 112.9 & 105.33 & 190 & 202 \\
\hline Maximum & 128.08 & 120.99 & 207 & 206.8 \\
\hline Mean & 121.483 & 112.928 & 198.5 & 3.112 \\
\hline Std. Deviation & 4.90973 & 4.72011 & 5.309 & 207 \\
\hline IQR & 8.9 & 6 & 5 & 198 \\
\hline Median & 122.095 & 113.23 & \multicolumn{2}{c}{$<0.001$} \\
\hline$p$-value & \multicolumn{2}{|l}{$<0.001$} & & \\
\hline
\end{tabular}

$121.4830 \pm 4.90973 \mathrm{~m}$ and non amblyopic eyes were $112.9280 \pm 4.72011 \mathrm{~m}$. Mean change in adults by using spectral domain Optical coherence tomography (OCT) and investigated whether 
these structures are affected in amblyopic eyes. However, this study does not include measurement of other structures such as choroidal thickness, mean macular or foveal volume, macular and optic disc morphology.

Walker et al13, enrolled 30 patients (60 eyes) adult population having anisometropic amblyopia. The average thickness of peripapillary retinal nerve fibre layer ( $\mathrm{pRNFL}$ ) was 90.6um ( $\mathrm{SD}=9.6$ ) of the amblyopic and 90.1um (SD=12.1um) fellow eye. The average thickness of macula was 260.1 um $(\mathrm{SD}=32.0 \mathrm{um})$ and $254.7 \mathrm{um}(\mathrm{SD}=32.5 \mathrm{um})$ of amblyopic and fellow eye respectively. Study determined that there was no significant difference of peripapillary and macular thickness of amblyopic and non amblyopic eyes. Wang et al14. Studied in 14 children, 5-10 years of age having anisometropic hyperopic amblyopia and concluded there is no difference inretinal nerve fibre (RNFL) thickness, central macular thickness (CMT) and total macular volume between amblyopic and non amblyopic fellow eyes. Another study conducted on strabismic amblyopia and refractive amblyopiaby Dickmann et al ${ }^{15}$. Which determined that thickness of mucula significantly increases only in the strabismic amblyopic group but did not find significant change in retinal nerve fibre layer (RNFL) thickness and foveal volume of amblyopic and non amblyopic fellow eyes of both groups (strabismic and refractive group). Yakar et $a l^{9}$, studied 30 adults who were hyperopic anisometropic amblyopia and compared central macular thickness (CMT) and peripapillary retinal nerve fibre layer ( $p$ RNFL) changes in amblyopic and fellow eyes. They observed a mean central macular thickness (CMT) in the amblyopic eyes (266.90 $\pm 23.22 \mathrm{~m}$ ) to be almost similar to fellow eyes $(263.90 \pm 22.84 \mathrm{~m})$ with an insignificant $p$-value $=$ 0.342. Also, the mean peripapillary retinal nerve fibre layer ( $p R N F L)$ thickness in amblyopic eyes (111.90 $\pm 12.9 \mathrm{~m})$, was comparable to fellow eyes $(109.70 \pm 9.42 \mathrm{~m})$, with the $p$-value again being insignificant $(p=0.621)$. Xu et al ${ }^{16}$, Celik et al 17 , carried out similar studies and found insignificant difference in pRNFL thickness, central macular thickness and macular volume in hyperopic anisometropic amblyopic population.

Contradictory to above results, Andalib et $a l^{18}$, studied two groups, anisometropic and strabismic amblyopic. Anisometropic amblyopia group results showed significant increase in the mean macular thickness while there was no significant change in peripapillary retinal nerve fibre layer (pRNFL) thickness in amblyopic and non amblyopic fellow eyes. Strabismic ambyopic group results showed no significant change in thickness of central macular thickness (CMT) as well asperipapillary retinal nerve fibre layer ( $p R N F L$ ) in amblyopic and nonamblopic fellow eyes. Pang et $a^{19}$, studied myopic anisometropic amblyopia, who have unilateral high myopia Huynh et al20. Studied in hypermetropic anisometropic amblyopic eyes. Both studies suggested increase in thickness of central macula (1mm diameter ring) and thickness decreases toward peripheral macula as compared to fellow eye in untreated amblyopic eyes. Al-Haddad et al21, found significantly increase in mean foveal volume in anisometropic amblyopia as well as in strabismic amblyopia patients. Several other studies, Szigeti et al22. Yalcin et al23, carried out which found significant increase of central macular thickness and insignificant changes in peripapillary retinal nerve fibre layer (pRNFL) thickness in anisometropic amblyopic patients.

Kim et al 24 , studied structural changes in deprivational amblyopic and non amblyopic fellow eyes. He found increase in the average thickness of retinal nerve fibre layer $(99.64 \pm 10.11 \mu \mathrm{m})$ of eyes having amblyopia when compared with non amblyopic fellow eyes $(97.28 \pm 12.34 \mu \mathrm{m})$ but change of thickness was not significant, only nasal retinal nerve fibre layer thickness of amblyopic $(75.84 \pm 19.22 \mu \mathrm{m})$ eyes were significant as compared with fellow eyes $(63.42 \pm 14.05 \mu \mathrm{m})$. He also found no significant difference of central macular thickness of amblyopic (237.05 \pm 37.74 $\mu \mathrm{m})$ and non amblyopic fellow eyes (226.67 \pm $34.71 \mu \mathrm{m})$. Wu et al 25 , studiedon 72 patients of hyperopic anisometropic amblyopia with average age of 5-16 years. He found significant increase 
in average peripapillary retinal nerve fibre layer (pRNFL) thickness in amblyopic eyes (113.9 \pm $7.2 \mu \mathrm{m})$ as compared to non amblyopic fellow eyes $(109.2 \pm 6.9 \mu \mathrm{m})$ with statistical significance $(p=0.02)$. Mean macular foveola thickness was $181.4 \pm 14.2 \mu \mathrm{m}$ in amblyopic eyes and $175.2 \pm$ $13.3 \mu \mathrm{m}$ in non amblyopic eyes with statistical significance $(p<0.01)$ but there was no significant difference in macular thickness of $1 \mathrm{~mm}, 3 \mathrm{~mm}$ or $6 \mathrm{~mm}$.

This study on anisometropic hyperopic amblyopia adults conducted on Pakistani population showed significant increase in peripapillary retinal nerve fibre layer ( $p$ RNFL) thicknessin amblyopic eyesas compare to non ambyolpic fellow eyes (table-II). On the other hand central macular thickness decreased in hyperopic anisometropic amblyopic eyes as compared to non amblyopic fellow eyes (table-II).

\section{CONCLUSION}

Central macular thickness (CMT) decreased, while peripapillary retinal nerve fibre layer (pRNFL) thickness increased in patients with anisometropic amblyopia. It is assumed that either structural changes i.e. central macular or retinal nerve fibre layer thickness play essential role in pathogenesis of amblyopia or these structural changes occurs after amblyopia in patients with anisometropic hyperopic amblyopia develops.

\section{CONFLICT OF INTEREST}

This study has no conflict of interest to be declared by any author.

\section{REFERENCES}

1. Siddiqui AH, Khan M, Hussain M, Mateen A, Khan A. Patterns of refractive error: prevalence and distribution of anisometropia. Pak J Surg 2017; 33(3): 226-29.

2. Akram SN, Ajmal M, Fatima K, Furqan A, Ali F. Anisometropic Amblyopic Patients. Prof Med J 2017; 24(03): 473-77.

3. Bogdanici ST, Costin D, Bogdanici CM. Quality of life for amblyopic children and their parents. Med-Surg J 2015; 119(1): 214-20.

4. Abdulghaffar AT, Al-Saadany A, Ibrahim AM. Optical coherence tomography-based comparison of retinal nerve fiber layer thickness and macular thickness in amblyopic and fellow eyes. Delta J Ophthalmol 2019; 20(2): 74-81.

5. Stanković B. Clinical aspects of different types of amblyopia. Vojnosanit Pregl 2011; 68(8): 696-98.

6. Li YP, Zhou MW, Forster SH, Chen SY, Qi X, Zhang HM, et al. Prevalence of amblyopia among preschool children in central south China. Int J Ophthalmol 2019; 12(5): 820-25.
7. Kantarci FA, Tatar MG, Uslu H, Colak HN. Choroidal and peripapillary retinal nerve fiber layer thickness in adults with anisometropic amblyopia. Eur J Ophthalmol 2015; 25(5): 437-42.

8. Kasem MA, Badawi AE. Changes in macular parameters in different types of amblyopia: optical coherence tomography study. Clinical Ophthalmology (Auckland, NZ) 2017; 11(1): 1407-16.

9. Yakar K, Kan E, Alan A, Alp MH, Ceylan T. Retinal nerve fibre layer and macular thicknesses in adults with hyperopic anisometropic amblyopia. J Ophthalmol 2015; 2015(1): 1-6.

10. Wong AM. New concepts concerning the neural mechanisms of amblyopia and their clinical implications. Can J Ophthalmol 2012; 47(5): 399-09.

11. Taşkıran AÇ. Retina and Optic Disc Characteristics in amblyopic and non-amblyopic eyes of patients with myopic or hyperopic anisometropia. Turk J Ophthalmol 2017; 47(1): 28-33.

12. Duman R. Characteristics of anisometropic patients with and without strabismus. Turk J Ophthalmol 2018; 48(1): 23-26.

13. Walker RA, Rubab S, Voll AR, Erraguntla V, Murphy PH. Macular and peripapillary retinal nerve fibre layer thickness in adults with amblyopia. Can J Ophthalmol 2011; 46(5): 425-27.

14. Wang BZ, Taranath D. A comparison between the amblyopic eye and normal fellow eye ocular architecture in children with hyperopic anisometropic amblyopia. J Am Assoc Pediatr Ophthalmol Strabismus 2012; 16(5): 428-30.

15. Dickmann A, Petroni S, Perrotta V, Salerni A, Parrilla R, Aliberti $\mathrm{S}$, et al. A morpho-functional study of amblyopic eyes with the use of optical coherence tomography and microperimetry. J Am Assoc Pediatr Ophthalmol Strabismus 2011; 15(4): 338-41.

16. Xu J, Lu F, Liu W, Zhang F, Chen W, Chen J. Retinal nerve fibre layer thickness and macular thickness in patients with esotropic amblyopia. Clin Exp Optom 2013; 96(3): 267-71.

17. Celik E, Çakır B, Turkoglu EB, Doğan E, Alagoz G. Evaluation of the retinal ganglion cell and choroidal thickness in young Turkish adults with hyperopic anisometropic amblyopia. Int Ophthalmol 2016; 36(4): 515-20.

18. Andalib D, Javadzadeh A, Nabai R, Amizadeh Y. Macular and retinal nerve fiber layer thickness in unilateral anisometropic or strabismic amblyopia. J Pediatr Ophthalmol Strabismus 2013; 50(4): 218-21.

19. Pang Y, Frantz KA, Block S, Goodfellow GW, Allison C. Effect of amblyopia treatment on macular thickness in eyes with myopic anisometropic amblyopia. Invest Ophthalmol Vis Sci 2015; 56(4): 2677-83.

20. Huynh SC, Samarawickrama C, Wang XY, Rochtchina E, Wong TY. Macular and nerve fiber layer thickness in amblyopia: the Sydney Childhood Eye Study. Ophthalmol 2009; 116(9): 1604-09.

21. Al-Haddad CE, Mollayess GM, Cherfan CG, Jaafar DF, Bashshur ZF. Retinal nerve fibre layer and macular thickness in amblyopia as measured by spectral-domain optical coherence tomography. Br J Ophthalmol 2011; 95(12): 1696-99.

22. Szigeti A, Tátrai E, Szamosi A, Vargha P, Nagy ZZ, Németh J, et al. A morphological study of retinal changes in unilateral amblyopia using optical coherence tomography image segmentation. PLoS One 2014; 9(2): e88363-71.

23. Yalcin E, Balci O. Peripapillary retinal nerve fiber layer and foveal thickness in hypermetropic anisometropic amblyopia. Clinical Ophthalmol (Auckland, NZ) 2014; 8(1): 749-5

24. Kim YW, Kim SJ, Yu YS. Spectral-domain optical coherence tomography analysis in deprivational amblyopia: a pilot study with unilateral pediatric cataract patients. Graefes Arch Clin Exp Ophthalmol 2013; 251(12): 2811-19.

25. Wu SQ, Zhu LW, Xu QB, Xu JL. Macular and peripapillary retinal nerve fiber layer thickness in children with hyperopic anisometropic amblyopia. Int J Ophthalmol 2013; 6(1): 85-89. 